\title{
Cytochrome-Oxidase Blobs in Cat Primary Visual Cortex
}

\author{
Kathryn M. Murphy, ${ }^{1}$ David G. Jones, ${ }^{2, a}$ and Richard C. Van Sluyters ${ }^{3}$ \\ ${ }^{1}$ McMaster University, Neural Organization and Plasticity Laboratory, Department of Psychology, Hamilton, Ontario \\ L8S 4K1, Canada, ${ }^{2}$ McGill University, Department of Electrical Engineering, Montreal, PQ H3A 2A7, Canada, and \\ ${ }^{3}$ University of California, School of Optometry, Berkeley, California 94720-2020
}

\begin{abstract}
Cytochrome-oxidase blobs are central to two of the most influential ideas in contemporary visual neurosciencecortical modularity and parallel processing pathways. In particular, the regular 2D array of cytochrome-oxidase-rich blobs in primate visual cortex is arguably the most compelling evidence for cortical modularity and has been hypothesized to mark a separate processing stream through the visual cortex. Although previously a variety of mammals have been studied, blobs have only been demonstrated in the visual cortex of primates, which has led to the conclusion that blobs represent a primate-specific feature of visual cortical organization. Here we demonstrate the presence of cytochrome-oxidase blobs In a nonprlmate species. Throughout the full tangential extent of layers IIIII in cat visual cortex the cytochrome-oxidase staining pattern is distinctly patchy, with the darkly stained blobs forming a regular 2D array. In addition, the blobs in cat visual cortex are functionally related to the underlying ocular dominance columns. The presence of cytochrome-oxidase blobs in the cat clearly demonstrates that they no longer can be considered a primate-specific feature of visual cortical organization.
\end{abstract}

[Key words: cytochrome-oxidase, blobs, cat, visual cortex, modules, ocular dominance]

Features of the mature visual cortex are distributed in a periodic fashion across its tangential extent forming the familiar modular structure of this cortical area (Hubel and Wiesel, 1962, 1974). A relatively simple histochemical staining technique for the mitochondrial enzyme cytochrome oxidase (CO) (Wong-Riley, 1979), produces a notably regular pattern of darkly stained patches in the upper layers of primate visual cortex (Horton and Hubel, 1981; Horton, 1984). The darker CO-stained patches are called blobs, and the lighter intervening regions, interblobs (Livingstone and Hubel, 1984a). The 2D pattern of CO blobs has become the most easily visualized neuroanatomical correlate of, and arguably the most compelling evidence for, cortical modu-

\footnotetext{
Received Aug. 17, 1994; revised Oct. 21, 1994; accepted Dec. 7, 1994.

This work was supported by NSERC grants (IJRF0124132, FQP004382.1 ICR0100439) to K.M.M., NIH grant (EY02193) to R.C.V.S., and NIH CORE Facilities Support Grant (EY03176). K.M.M. is an NSERC University Research Fellow and an Alfred P. Sloan Research Fellow. We are especially indebted to Drs. Michael P. Stryker, Steven W. Zucker, and Jonathan Horton for their helpful discussion of these data.

Correspondence should be addressed to Dr. Kathryn M. Murphy, McMaster University, Neural Organization and Plasticity Laboratory, Department of Psychology, 1280 Main Street West, Hamilton, Ontario L8S 4K1, Canada.

aPresent address: McMaster University, Department of Computer Science, 1280 Main Street West, Hamilton, Ontario L8S 4K1, Canada.

Copyright $(1995$ Society for Neuroscience $\quad 0270-6474 / 95 / 154196-13 \$ 05.00 / 0$
}

larity in primate visual cortex. The distribution of CO blobs has provided a robust, albeit opportunistic, anatomical marker with which to correlate the distribution of physiological properties in the visual cortex, the array of local cortical circuits, and the arrangement of corticocortical connections. These studies have allowed the formation of links between physiological properties and the underlying anatomy of both the modular organization of the primary visual cortex and the streaming of visual information into parallel processing pathways (Zeki, 1975; Livingstone and Hubel, 1983, 1984a; DeYoe and Van Essen, 1985, 1988; Shipp and Zeki, 1985; Van Essen et al., 1992).

In the monkey, blobs lie at evenly spaced intervals along ocular dominance columns (Fitzpatrick and Diamond, 1980; Hendrickson et al., 1981; Horton, 1984) and are linked by a latticework of blob-specific horizontal connections (Livingstone and Hubel, 1984b). Cells within blobs tend to be less binocular and less selective for orientation, but more color selective, than cells in interblob regions (Livingstone and Hubel, 1984a), and projections from blob or interblob regions to higher cortical areas exhibit a certain degree of segregation (Zeki, 1975; Livingstone and Hubel, 1983, 1984a, 1987a,b). Hence, in addition to being an impressive anatomical demonstration of cortical modularity, the $\mathrm{CO}$ blobs have proven to be a key feature for understanding the parcelling and streaming of functions within the primate visual system.

Although previously a variety of mammals have been studied (e.g., Wong-Riley, 1979; Horton, 1984; Price, 1985), blobs have only been demonstrated in the visual cortex of primates, which has led to the widely accepted notion that blobs represent a primate-specific feature of visual cortical organization. The lack of evidence for $\mathrm{CO}$ blobs in other mammals resulted in primates becoming the animal model for studies of parallel processing streams and frustrated attempts to formulate general models for the functional organization of modules in the mammalian visual system. For example, the pattern of $\mathrm{CO}$ staining in the cat visual cortex has been described as uniform within the supragranular layers (e.g., Wong-Riley, 1979; Horton, 1984; Price, 1985), thereby raising the possibility of a significant interspecies difference, even at this relatively early stage in the visual pathway, in the other higher mammal commonly studied in visual neuroscience. Thus, although other features such as ocular dominance columns appear to arise from similar constraints in cats and monkeys (Jones et al., 1991), the apparent lack of blobs in the cat became an impediment for integrating information gleaned from experiments using the cat model into an overall understanding of the significance of cortical modularity for visual perception.

In our previous studies of the anatomical organization of oc- 
ular dominance in the cat visual cortex; we came to appreciate the insights that can be gained when the overall organization of this pattern is examined in large, tangential sections cut from an unfolded and flattened cerebral hemisphere (Anderson et al., 1988; Murphy et al., 1989). For example, viewed in this way, the ocular dominance pattern is easily recognized as a two-dimensional network of branching, beaded bands that are not oriented along any particular axis. In our experience, examination of sections cut tangential to the surface of the unfolded and flattened cortex provides the most sensitive technique for resolving virtually any anatomical pattern that is arrayed parallel to the cortical laminae. This is especially true when the histological image of the anatomical pattern is of low contrast. For this reason, along with the possibility that blobs might be spatially related to the underlying pattern of ocular dominance columns in the cat, we were encouraged to reexamine this issue in tangential sections cut from unfolded and flattened visual cortex. Here, we demonstrate that cytochrome-oxidase blobs are, indeed, a feature of primary visual cortex in nonprimate species.

Preliminary reports of this discovery have included some of these data (Murphy et al., 1990, 1991a,b).

\section{Materials and Methods}

Animals and surgical procedures. A total of 10 adult cats were used to study the organization of $\mathrm{CO}$ blobs in the visual cortex. The cats were placed into one of four experimental groups; no manipulations $(n=3)$, unilateral eye injection with wheatgerm agglutinin conjugated to horseradish peroxidase (WGA-HRP) $(n=3)$, unilateral retina lesion $(n=$ 2 ), and unilateral retinal lesion plus WGA-HRP injection into the fellow eye $(n=2)$. The complete pattern of cortical ocular dominance was revealed following unilateral intraocular injections of the anterograde transneuronal tracer WGA-HRP (Sigma, St. Louis, MO) (5\% in saline), using previously described procedures (Anderson et al., 1988). To further examine the relationship of the CO blobs to cortical ocular dominance columns, a complete retinal lesion of one eye was performed. Aseptic surgical techniques, gaseous anesthetic $(0.5-5.0 \%$ halothane in $67 \% \mathrm{~N}_{2} \mathrm{O} / 33 \% \mathrm{O}_{2}$ ), and postsurgical analgesics were used for the retinal lesion procedure. A circumlimbial incision of the cornea was made, exposing the iris and lens. Using light suction, the lens was extracted through the pupil, taking care to not tear the iris or ciliary body. The vitreous humor was aspirated, then the whole retina was swabbed free from the eyecup. Saline-and-thrombin-soaked Gelfoam was used to pack the eyecup tightly and the circumlimbial incision was sutured closed. A broad spectrum ophthalmic antibiotic was applied to the front of the closed eyecup, then the lid margins were sutured together for about $7 \mathrm{~d}$ to protect the ocular incision while it healed. Postlesion survival periods ranged from 7 to $90 \mathrm{~d}$.

Histology. Animals were euthanized with a lethal injection of Nembutal $(150 \mathrm{mg} / \mathrm{kg}$, i.p.) then perfused transcardially with saline $(1-1.51$ at $45^{\circ} \mathrm{C}$ ) followed by $4 \mathrm{~min}$ of fixation with $2 \%$ glutaraldehyde in 0.1 $\mathrm{M}$ phosphate buffer $\left(4^{\circ} \mathrm{C}\right)$. This perfusion protocol was optimized for the process of unfolding and flattening the cortex; glutaraldehyde fixes the gray matter while leaving the white matter relatively unfixed. The brain was removed from the cranium and the cerebral hemispheres were resected for unfolding and flattening (Olavarria and Van Sluyters, 1985). The block of brain containing the dLGNs was placed in $30 \%$ sucrose, $2 \%$ glutaraldehyde in $0.1 \mathrm{M}$ phosphate buffer and refrigerated $\left(4^{\circ} \mathrm{C}\right)$. During the unfolding and flattening of the cortex the hemispheres were kept wet with $2 \%$ glutaraldehyde while the white matter was carefully excised. After removal of the white matter, the intrinsic curvature in the sheet of gray matter was relieved by making a medial and a lateral cut, thereby allowing the visual cortex to lie flat. It is important to note that at this stage in the unfolding and flattening proccdure the cortical tissuc was well fixed, and these cuts in the tissue were essential to prevent tearing or possible distortion of the visual cortex during flattening. Once flattened, the hemispheres were postfixed with $2 \%$ glutaraldehyde, $30 \%$ sucrose in phosphate buffer $\left(4^{\circ} \mathrm{C}\right)$ for up to $6 \mathrm{hr}$, then transferred to $30 \%$ sucrose in phosphate buffer $\left(4^{\circ} \mathrm{C}\right)$ and stored overnight. The following day the unfolded and flattened cortices were cut tangential to the pial surface $(50 \mu \mathrm{m})$ on a freezing microtome and the sections were collected in phosphate buffer. For those cases where both $\mathrm{CO}$ and labeled ocular dominance columns were studied, all the supragranular sections to a depth of $550 \mu \mathrm{m}$ were stained for $\mathrm{CO}$, then alternate sections were either stained for $\mathrm{CO}$, or reacted with TMB to reveal the labeled ocular dominance columns.

The sections for $\mathrm{CO}$ staining were mounted onto gelatin-coated glass slides, allowed to air dry, and then reacted (5-7 hr incubation at $\left.40^{\circ}\right)$ following the standard $\mathrm{CO}$ histochemical procedure used by Horton (1984). All sections from a hemisphere were reacted together for the same incubation period. The dLGNs were sectioned coronally on a freezing microtome $(50 \mu \mathrm{m})$, mounted onto slides, and air dried, then reacted for $\mathrm{CO}$ ( $2-4 \mathrm{hr}$ incubation). Stained slides were dehydrated and defatted, coverslipped with DPX (Aldrich, Milwaukee, WI), and allowed to dry before photographing.

Sections reacted to reveal the labeled pattern of ocular dominance columns were collected in phosphate buffer, washed in three changes of buffer, then reacted with the chromogen tetramethylbenzidine (Sigma, St. Louis) using the Mesulum histochemical procedure as modified by Gibson et al. (1984). This modification keeps the A and B parts separate and continuously filters the B solution, thereby minimizing the amount of precipitate and resulting in very high sensitivity for revealing transneuronally transported WGA-HRP. Once any bluish precipitate was washed out in the postreaction rinse, the sections were mounted onto gelatin-coated glass slides and air dried. The sections werc dehydrated and defatted, then coverslipped using DPX.

Tangential analysis. The relationship between the CO blobs and ocular dominance columns was studied in two groups of animals: one in which ocular dominance columns were visualized following an eye injection of WGA-HRP, and a second where one eye was injected with WGA-HRP while retinal activity was silenced in the fellow eye by lesioning the whole retina. In both of these groups, the tangential relationship of the blobs in layers II/III was compared with that of the ocular dominance pattern from layer IV. Using radial blood vessels to manually align sections through layers II/III and IV, a region with distinct blobs was analyzed. These successive tangential sections through layers II/III and IV were input to a computer system (SUN4) by imaging with a color CCD camera (Sony, XC-711) connected to a large-format macrophotography system and frame-grabber board (Parallax Graphics, $\mathrm{XVideo} / \mathrm{RGB}$ ). The low magnification $(0.7-5.0 \times)$, good color resolution of the imaging system, and ability to store and view multiple aligned raw images, were helpful in locating the CO blobs. First, blobs were visualized and marked with a cross using the computer system to view the aligned raw images from all supragranular $\mathrm{CO}$-stained sections in rapid succession, in a manner akin to focusing up and down through the sections. Next the resulting pattern of crosses was overlaid onto aligned serial images of the ocular dominance pattern representing the full thickness of layer IV; the crosses were then color coded such that crosses coincident with the labeled ocular dominance pattern were coded red, and crosses coincident with the unlabeled ocular dominance regions were coded green. During the analysis, the positions of the blobs were localized without any prior knowledge of the underlying ocular dominance pattern, and this was done independently by two obscrvers. This analysis was performed on rectangular regions within area 17 , varying in size from $4.5 \times 3 \mathrm{~mm}$ to $10 \times 7 \mathrm{~mm}$. The technique for serially viewing all of the CO-stained sections on the computer aids in the analysis of these data in two ways. First, the third dimension is included, thereby eliminating the problem of missing blobs in a single $2 \mathrm{D}$ section because regions had dipped out of the plane of section. Second, viewing successive aligned sections improves the signal-tonoise, since the spatial location of the blobs is consistent through many images, while nonspecific variations in staining show no spatial correlation across sections. Thus, the apparent contrast of the blobs is improved. In addition, a second analysis was performed with aligned single sections, one section from layers II/III stained for $\mathrm{CO}$ with obvious blobs, and the other section from layer IV reacted to reveal the pattern of ocular dominance following an cyc injection. Each image was thresholded at midluminance to produce binarized images; the binarized blob and ocular dominance images were added to generate a color-coded image of the relationship between blobs and ocular dominance.

\section{Results}

When single tangential sections through the posterior neocortex of the cat are stained for $\mathrm{CO}$, the ovate, two-dimensional shape of areas 17 and 18 stands out as a region that generally stains 

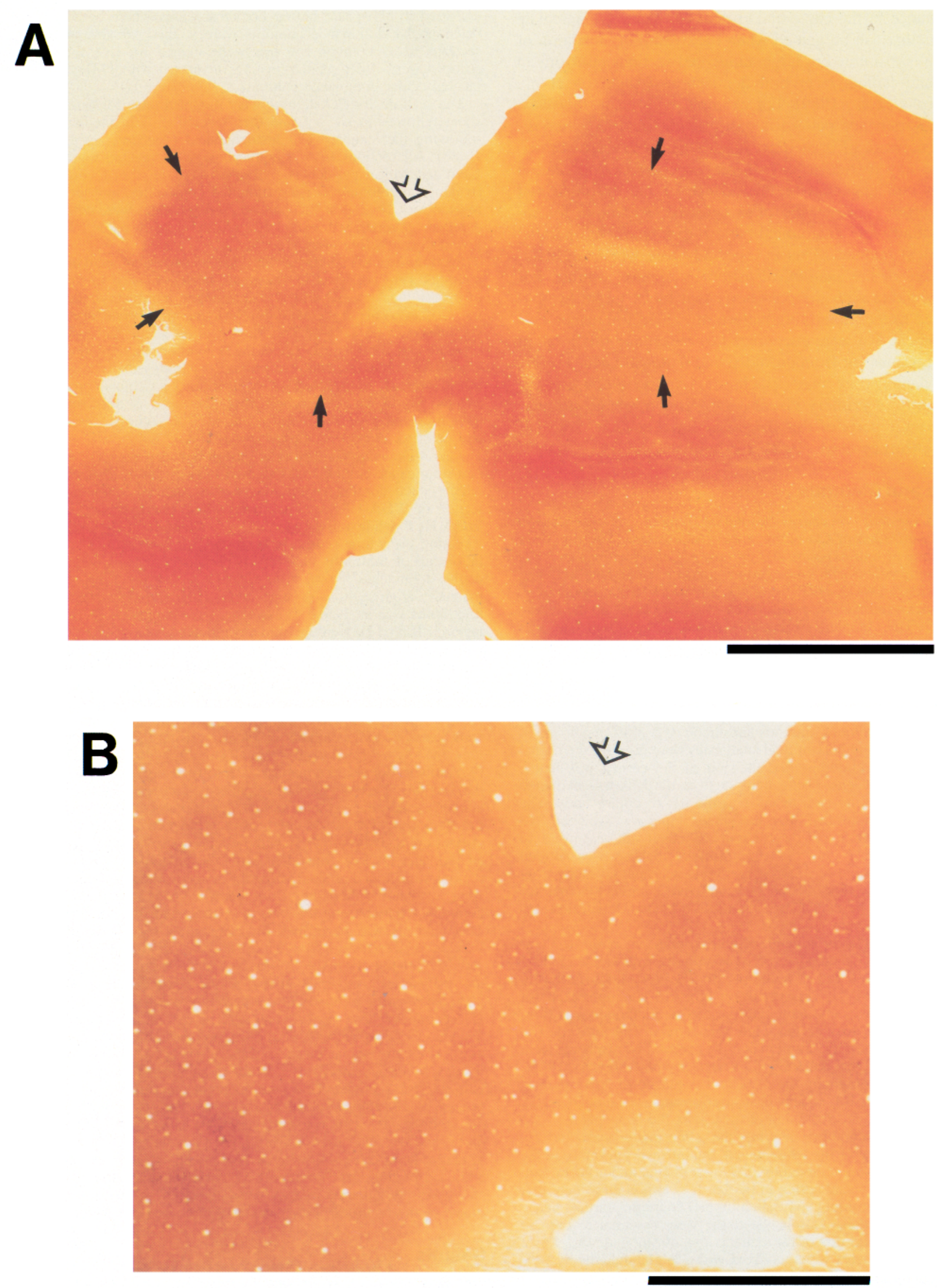

Figure 1. A, A single tangential section $(4.2 \times 3 \mathrm{~cm}, 50 \mu \mathrm{m}$ thick), through supragranular layers from the unfolded and flattened posterior neocortex of the cat, stained for cytochrome oxidase. The complete $2 \mathrm{D}$ extent of area 17 is visible in this section as the darker stained 

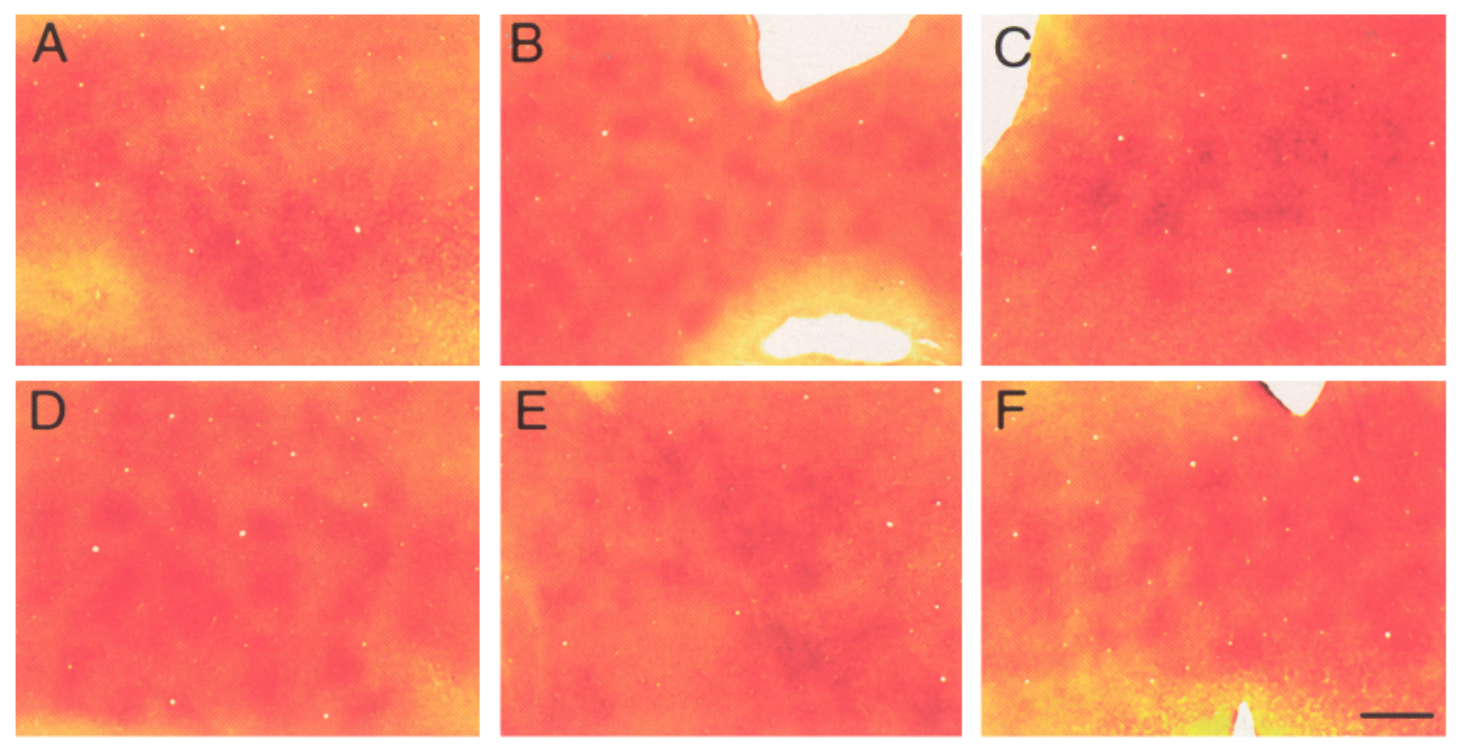

Figure 2. The six panels in this figure show the pattern of $\mathrm{CO}$ staining observed in the supragranular layers of area 17 from each of the six normally reared cats studied in the present experiment. Scale bar, $1 \mathrm{~mm}$.

more darkly than the surrounding cortical areas. Closer inspection of the staining pattern reveals that within area 17 of the cat cortex the staining pattern is nonuniform in certain sections. Throughout the full extent of area 17 the staining pattern in tangential sections passing through layers II-III is distinctly patchy $-\mathrm{CO}$ blobs are, indeed, present in supragranular layers (Fig. 1A). The nature of these patches of darker $\mathrm{CO}$ staining is even more apparent at slightly higher magnification (Fig. 1B). The blobs were generally somewhat more distinct in the region of area 17 in which the central part of the visual field is represented (see Fig. $1 B$ ) than in the region representing the periphery of the visual field. This tangential variation in the qualitative appearance of the blobs was a consistent feature of all hemispheres studied and resembles tangential variations present in the cat ocular dominance pattern (Anderson et al., 1988; Murphy et al., 1989). Further analyses are confined to the more central visual field representation $\left(<10^{\circ}\right)$ within area 17 .where both the blobs and ocular dominance patterns are most easily distinguished.

Blobs were observed in the primary visual cortex in both hemispheres of all cats studied. Figure 2 shows the appearance of the blobs observed in each of the six normal cats studied. It is apparent from comparison of the pattern of $\mathrm{CO}$ staining shown in the panels of Figure 2 that the shape, size, and spacing of the blobs appears similar in all of the cats studied. In primate cortex, $\mathrm{CO}$ staining is nonuniform in a number of extra-striate cortical areas, most notably V2, which has an arrangement of thick and thin darkly stained stripes (Horton, 1984). In our initial examinations of cat area 18 we have not observed any consistent pat- terning of the staining in this area. This may reflect the fact that unlike the primate, area 18 in the cat receives some direct input from LGN Y-cells, or it may simply be that the incubation times used to achieve optimal staining of area 17 are not appropriate for staining area 18. It is apparent in the CO-stained tangential sections through areas 17 and 18 (see Fig. 1A) that, as has been reported using coronal sections (Price, 1985), there is no abrupt change in $\mathrm{CO}$ staining at the area $17 / 18$ border and that the dramatic change in the density of staining occurs more lateral at the area $18 / 19$ border.

A patchy pattern of $\mathrm{CO}$ staining in layer IV of cat visual cortex had been reported previously only following disruption of normal binocular visual experience (Wong-Riley, 1979; Wong-Riley and Carroll, 1984). If an abnormality associated with one eye resulted in the patchy pattern of $\mathrm{CO}$ staining in the cortex observed in the present study, then it also should have resulted in reduced staining of the layers of the dorsal lateral geniculate nucleus (dLGN) that receive their input from that eye (Wong-Riley and Riley, 1983; Kageyama and Wong-Riley, 1986). Examination of dLGN sections reacted for CO revealed that the staining was of comparable density in the right- and left-eye layers of the normally reared cats (Fig. 3A). This is contrasted with the staining pattern found following monocular retinal lesion where there was a reduction in the density of staining in the dLGN laminae subserving the lesioned eye (layer A1 in Fig. 3B).

Having established the presence of blobs in the cat visual cortex, some of their anatomical characteristics were examined. The radial extent of the blobs was assessed by using blood vessel

$\leftarrow$

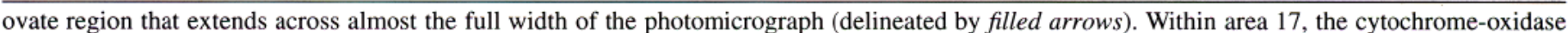

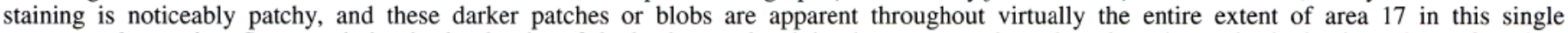

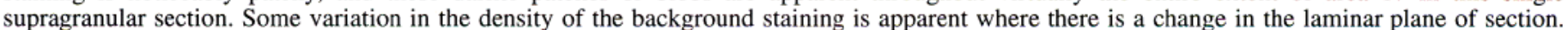

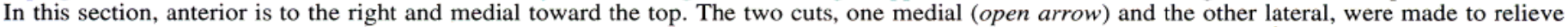

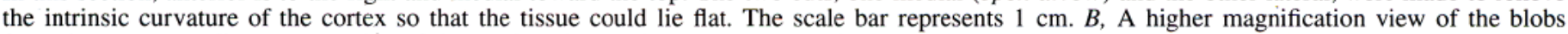

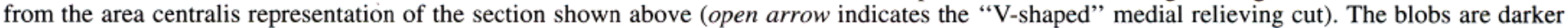

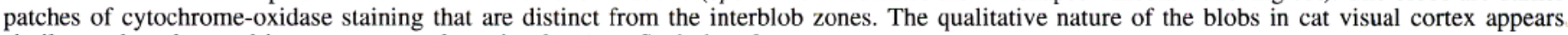
similar to that observed in macaque monkey visual cortex. Scale bar, $2 \mathrm{~mm}$. 

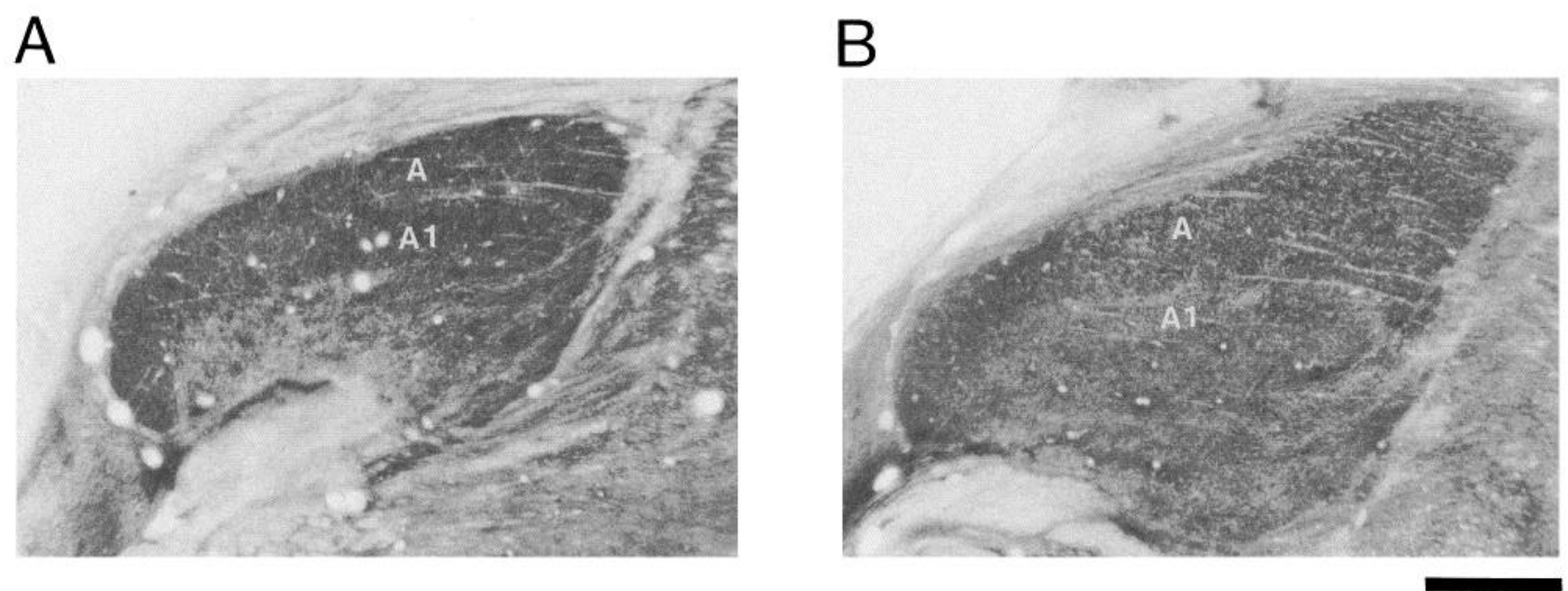

Figure 3. A, The pattern of CO staining in the dLGN of a normally reared cat. The A and Al laminae are darkly stained and of comparable density. $B$, CO-stained section from the dLGN ipsilateral to an eye with a complete retinal lesion. The density of staining is notably lighter in the lamina that receives input from the lesioned retina (A1 layer) than the layer that receives input from the intact eye (A layer). In these sections, dorsal is to the top and medial to the right. Scale bar, $1 \mathrm{~mm}$.

landmarks to align serial tangential sections and following the staining pattern for individual blobs through adjacent sections. Typically, blobs are first faintly visible at a depth of about 250$300 \mu \mathrm{m}$ below the pial surface (Fig. 4A). The blobs become progressively darker, reaching their maximum density at about $450-500 \mu \mathrm{m}$ below the surface (Fig. 4C). Beneath this depth, the blobs rapidly merge toward a uniformly dense staining pattern within layer IV, so that by about $550-600 \mu \mathrm{m}$ below the pial surface (Fig. $4 E$ ) they are no longer visible. Often the blob pattern is only easily discernible where the contrast between blob and interblob regions is the highest (i.e., about $450 \mu \mathrm{m}$ below the pial surface). This latter finding underscores the importance of carefully unfolding and flattening the visual cortex in order to obtain tangential sections with large regions that pass through the portion of layers II-III where blobs are most easily discriminated. Compiling the radial changes observed from several animals reveals that blobs are largely confined to layers IIIII of cat visual cortex and that their total radial extent is approximately $250-300 \mu \mathrm{m}$.

We extended the radial analysis of the blobs in the cat to include comparison with the location of the geniculocortical terminations, labeled following an eye injection, in comparable series of tangential sections from an earlier study (Anderson et al., 1988). Since all sections in this and the earlier study were from unfolded and flattened cortices cut tangentially at a thickness of $50 \mu \mathrm{m}$, it was possible to compare across animals and begin to analyze the depth below the pial surface where either blobs or geniculocortical inputs are located. As described above, the radial location of the blobs is from a depth of about $250-500 \mu \mathrm{m}$ and geniculocortical afferents were seen to occupy the region extending from about 450 to $850 \mu \mathrm{m}$ below the pial surface (Anderson et al., 1988). Clearly, most of the radial extent of the blobs must be superficial to the geniculocortical afferents, and the laminar positions where each of these patterns is most distinct are not coincident. However, at their deepest extent the blobs may overlap the zone of geniculocortical input (LeVay and Gilbert, 1976; Leventhal, 1979).

It should be recognized that the presence of $\mathrm{CO}$ blobs in the monkey was a mere curiosity until they were linked with other features of cortical organization. One of the first relationships to be examined in monkeys was that between the blobs and cortical ocular dominance columns, where it was shown that blobs are both spatially correlated with ocular dominance columns and functionally eye specific (Fitzpatrick and Diamond, 1980; Hendrickson et al., 1981; Horton, 1984). In the present study, the spatial relationship was examined in an additional three normally reared adult cats in which the tangential patterns of both $\mathrm{CO}$ blobs and ocular dominance columns in area 17 were visualized within the same hemisphere. An example of an analysis performed to examine whether there is a simple spatial relationship between the position of the $\mathrm{CO}$ blobs and the ocular dominance patterns is shown in Figure 5, where the tangential location of blobs in all sections through layers II/III were marked (Fig. 5A; see Materials and Methods for details) and aligned using radial blood vessels with the underlying WGA-HRP labeled ocular dominance pattern from layer IV. The locations of the blobs were then color coded to reflect whether a blob was associated with a labeled (red crosses) or an unlabeled (green crosses) ocular dominance column. In the color-coded map (Fig. 5B), it appears that there is a relatively even mixture of blobs that are associated with either the ipsilateral (labeled) eye's ocular dominance columns (red crosses), or the presumed contralateral (unlabeled) eye's columns (green crosses). The presence of both red and green crosses in this analysis indicates that the blobs do not simply reflect the pattern of one eye's inputs. The spatial locations of these red and green crosses, however, are not positioned solely above the centers of the underlying ocular dominance columns. Thus, an additional analysis of this spatial relationship was done to assess the areal extent of blobs with respect to the pattern of ocular dominance. From this analysis we could visualize whether blobs located near an ocular dominance boundary are encompassed by or straddle ocular dominance domains. Regions from single supragranular sections with distinct blobs (Fig. 6A, red blobs) and a layer IV section reacted to reveal the pattern of inputs from one eye (Fig. $6 B$, green labeled ocular dominance) were aligned, binarized, color coded, and then added together (Fig. $6 C$; see Materials and Methods for details). Clearly, from this analysis (Fig. 6C) some blobs are situated largely within the unlabeled (largely red blobs) regions of the ocular dominance pattern, while others are encompassed by the 

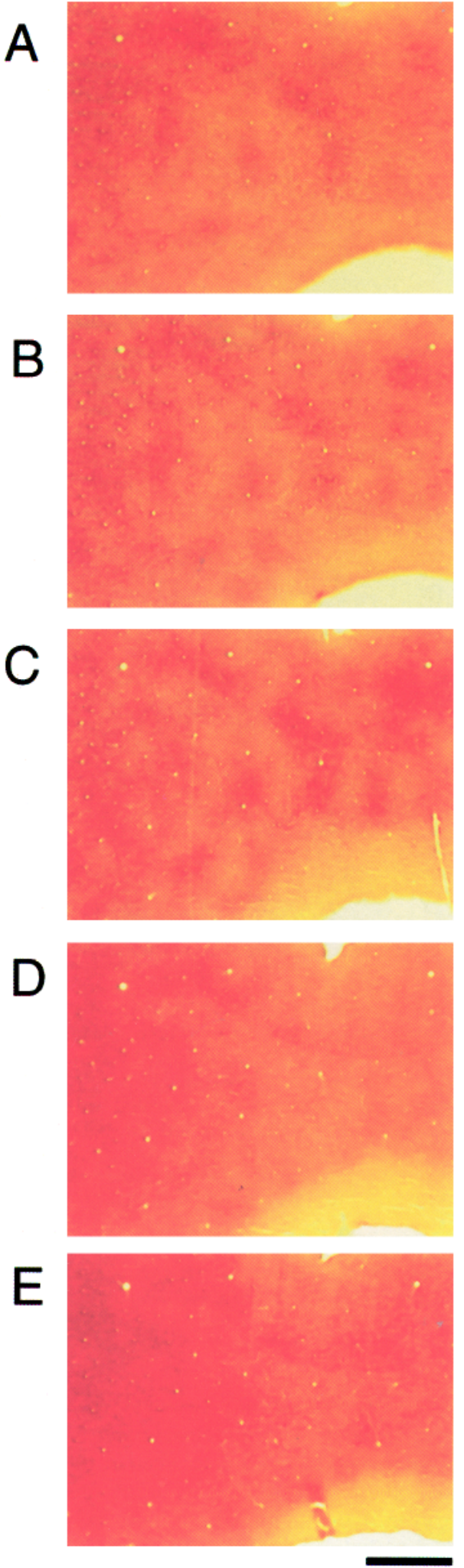

Figure 4. Radial changes in the pattern of $\mathrm{CO}$ staining through layers II/III $(A-C)$ and into upper layer IV $(D-E)$. The sections were aligned, using the radial blood vessels, to allow for comparison of the $\mathrm{CO}$ staining pattern in these layers. The pattern of CO staining $(A) 250 \mu \mathrm{m},(B)$ $350 \mu \mathrm{m}$, and $(C) 450 \mu \mathrm{m}$, below the pial surface is consistently patchy. Using the radial blood vessels as landmarks to align sections, it is clear that the dark patches of $\mathrm{CO}$ staining are in register radially and form a columnar structure. The subsequent panels show $(D)$ the pattern of CO staining at the transition between layers II/III and layer IV $(500 \mu \mathrm{m}$ labeled (yellow blobs) zones, and some straddle the labeled and unlabeled domains (red and yellow). The results of these two analyses indicate that in the cat the spatial relationship between blobs and ocular dominance does not follow the same simple pattern observed in primates (see Discussion). However, the finding in normal cats that some blobs do overlie either the labeled or unlabeled aspects of the underlying ocular dominance pattern leaves open the possibility that blobs may be functionally eye specific.

At this point, the relationship between ocular dominance and blobs had only been examined as a simple spatial correlation from which it is tenuous to either draw or eliminate possible functional relationships. Therefore, we challenged the hypothesis that blobs may be functionally eye specific by using one of the techniques employed in previous primate studies to address this issue (e.g., Horton, 1984), that of silencing the activity from one eye. The notion of a functional relationship was tested by examining the arrangement of blobs with ocular dominance columns in four cats that were monocularly enucleated as adults (Fig. 7). This manipulation reduces $\mathrm{CO}$ staining in the layers of the dLGN (Fig. 3B) and in the cortical ocular dominance columns corresponding to the enucleated eye. If the blobs are not eye specific, then one would expect their tangential arrangement to be unchanged by this manipulation, and the analysis of their relationship to ocular dominance columns would still have red and green crosses. Conversely, if the blobs are eye specific then, as has been demonstrated in the monkey (Horton, 1984), there should be a significant reduction in staining of blobs overlying the enucleated eye's ocular dominance columns, possibly to the point where those blobs are no longer discernible, and the analysis would result in crosses of only one color. To ensure that a reduction in contrast of some blobs did not bias this analysis against finding blobs over unlabeled regions of the ocular dominance pattern, care was taken to mark every region of denser $\mathrm{CO}$ staining that may possibly represent a blob. A color-coded map (Fig. $7 B$ ) of the relationship between the 2D arrangement of $\mathrm{CO}$ blobs and ocular dominance columns following monocular enucleation shows that blobs now are almost exclusively associated with the remaining eye's ocular dominance columns-there are only red crosses in this analysis. Further analysis of the relationship between blobs and ocular dominance following enucleation reveals that blobs are virtually encompassed (yellow regions) by the underlying ocular dominance pattern (Fig. 8), such that very little red remains when the binarized blobs and ocular dominance patterns are added. This contrasts with the arrangement found in nonlesioned cats, where many blobs overlie the unlabeled regions of the ocular dominance pattern (see Figs. $5 B, 6 C$ ). Following a complete retinal lesion, we could find no blobs that appear to be above the unlabeled regions of the ocular dominance pattern, the presumed monocular aspects of the lesioned eye's ocular dominance columns. The results of these two analyses (Figs. $7 B$ and $8 C$ ) solidifies the argument for a functional relationship between blobs and ocular dominance in the cat. Furthermore, the remaining $\mathrm{CO}$ blobs in layer II/III were patchy (see Figs. $7 A, 8 A$ ) and so it was not the case that the pattern of $\mathrm{CO}$ staining in the supragranular layers merely reflected the complete pattern of geniculocortical affer-

\section{$\leftarrow$}

below the pial surface), and (E) layer IV ( $550 \mu \mathrm{m}$ below the pial surface) where the $\mathrm{CO}$ staining pattern becomes dark and relatively uniform. Scale bar, $1 \mathrm{~mm}$. 

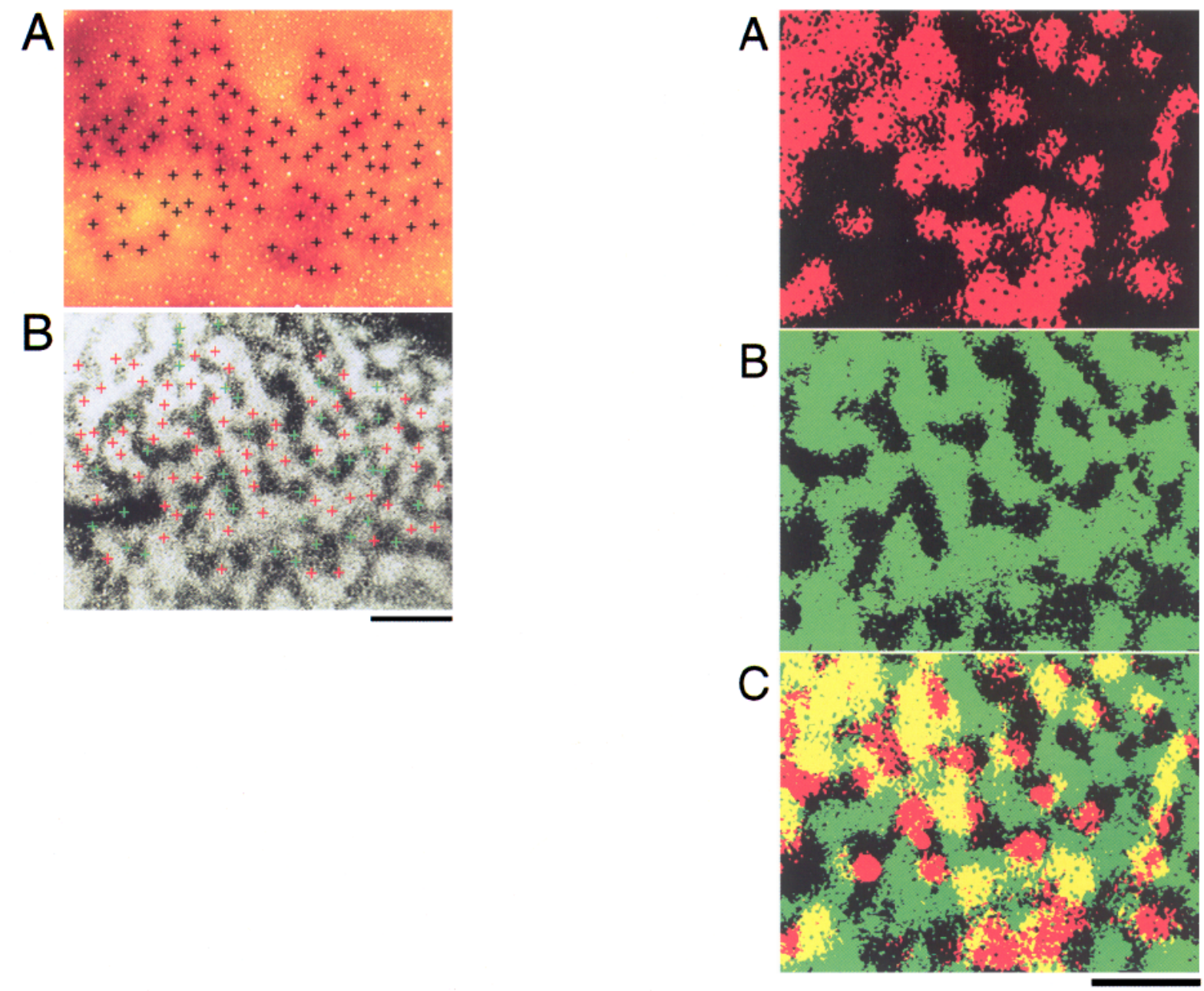

Figure 5 (left). Using radial blood vessels to align a series of CO-stained sections with those reacted to reveal the pattern of ocular dominance columns, a region with distinct blobs was overlaid onto the underlying ocular dominance pattern. $A$, In this 10 by $7 \mathrm{~mm}$ portion of a tangential section from layers II-III ( $450 \mu \mathrm{m}$ below the pial surface) crosses mark the location of blobs. The blobs were located by comparison with the adjacent cytochrome-oxidase stained sections and in this example the range of center-to-center spacing is approximately $350-1000 \mu m$. $B$, This image shows the relationship that is revealed by overlaying the crosses onto the underlying ocular dominance pattern revealed following intraocular injection of the transneuronal tracer WGA-HRP. In this dark-field view of a section from a depth of $750 \mu \mathrm{m}$ (i.e., $300 \mu \mathrm{m}$ below that in $B$ ), the light bands represent the arrangement of the geniculocortical inputs from the injected ipsilateral eye. Where the blobs are coincident with the labeled ocular dominance columns, the crosses are color coded red, and where they are coincident with the unlabeled ocular dominance regions the crosses are coded green. Similar results were obtained when the hemisphere contralateral to an injected eye was examined. Scale bar, $2 \mathrm{~mm}$.

Figure 6 (right). Visualization of the relationship between aligned regions of $(A) \mathrm{CO}$ blobs $($ red $)$ in single sections through layers II/III, and $(B)$ ocular dominance (green) in layer IV revealed following an eye injection with WGA-HRP in a normally reared cat. Images of the CO staining and ocular dominance were thresholded at midluminance then added to generate a color-coded image. $C$, CO blobs over the labeled eye's inputs are shown in yellow, blobs over the unlabeled regions are shown in red. Scale bar, $1 \mathrm{~mm}$.

ents subserving the surviving eye (see Figs. $7 B, 8 B$ ). Thus, the functional relationship between blobs and ocular dominance columns in the cat seems similar to that found in the monkey; in both species, blobs exhibit eye specificity.

Parenthetically, no previous study in the cat had demonstrated that the pattern of CO staining observed in layer IV following disruption of retinal activity reflects the remaining eye's ocular dominance columns. In this study, the combination of a retinal lesion, $\mathrm{CO}$ staining, and an eye injection of a transneuronal tracer to visualize the pattern of ocular dominance in layer IV, answered this question. Images of adjacent sections from layer IV, either stained for $\mathrm{CO}$ or reacted to reveal the pattern of the surviving eye's inputs, were added together to determine the relationship between these patterns. A negative contrast image of CO staining (dense CO staining is white) was added with the dark-field image of the ocular dominance pattern. With this analysis, it is straight forward to visualize whether the labeled ocular dominance and dense $\mathrm{CO}$-staining patterns are in register or complementary. In the situation where these two patterns are in register, the resulting image would appear very similar to each of the component images. On the other hand, if the ocular dominance and $\mathrm{CO}$ images are complementary, then the resulting image would be uniformly white. The visual cortices examined in this experiment showed good agreement in layer IV between the pattern of $\mathrm{CO}$ staining (Fig. 9A) and the pattern of ocular dominance columns labeled following injection of the surviving eye (Fig. 9B). Since the anatomical features present in CO staining (Fig. 9A) and labeled ocular dominance (Fig. 9B) persist in 

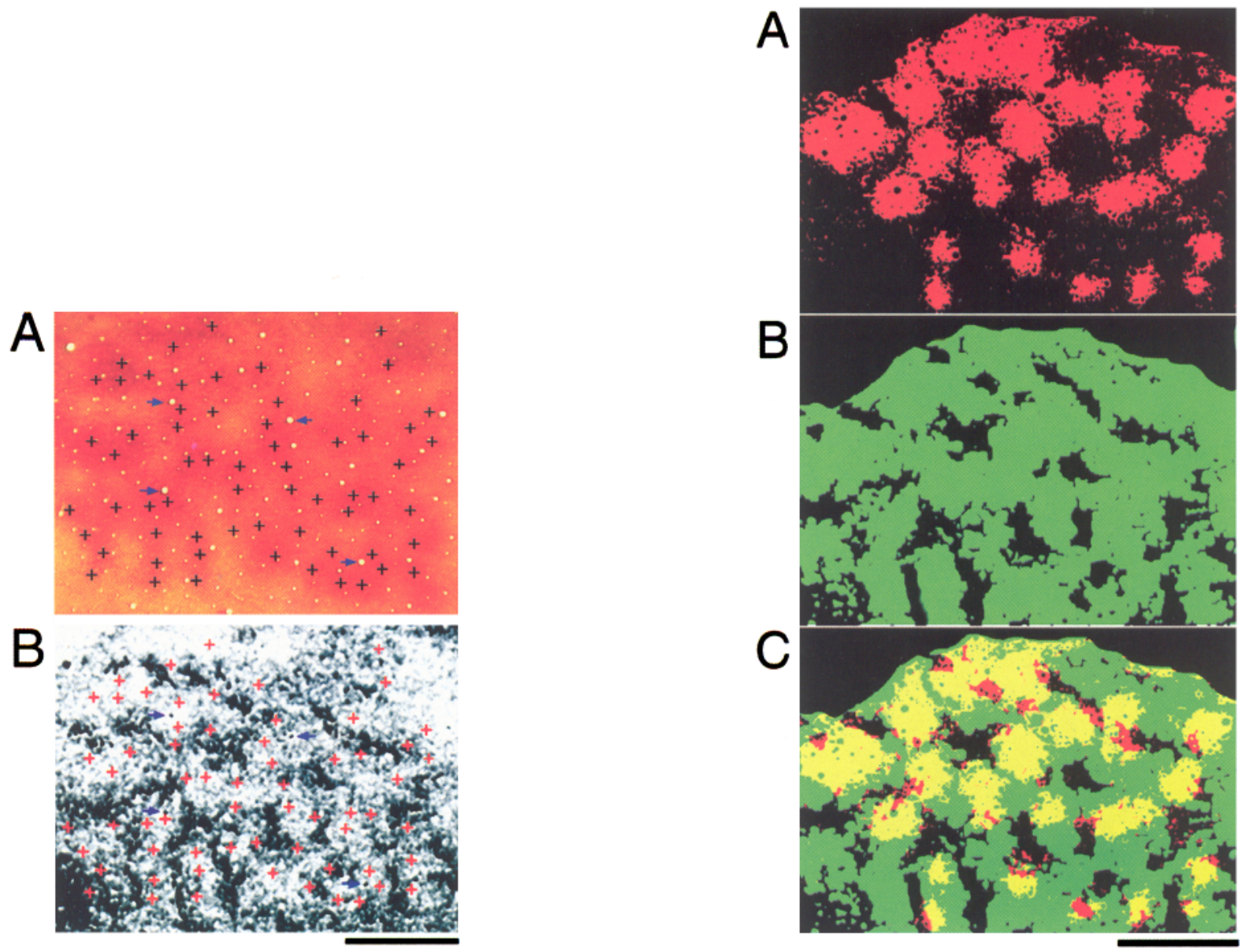

Figure 7 (left). The relationship between $\mathrm{CO}$ blobs and the underlying ocular dominance pattern is shown following 2 weeks survival after monocular enucleation. $A$, Cytochrome-oxidase blobs are apparent in this 7 by $5 \mathrm{~mm}$ portion of a tangential section through layers II-III. $B$, By comparison with adjacent CO-stained sections, the blobs in $A$ are marked with crosses. The arrows indicate some of the radial blood vessels used for alignment. $B$, A color-coded map of the relationship of blobs to the underlying ocular dominance columns revealed following an eye injection of WGA-HRP, prepared according to the methods described in Figure 5. The light bands represent the geniculocortical inputs from the remaining eye. As indicated by the red crosses, after monocular enucleation, all of the blobs in layers II-III overlie the remaining eye's ocular dominance columns. Scale bar, $2 \mathrm{~mm}$.

Figure 8 (right). Following complete monocular retinal lesion, the arrangements of $(A)$ CO blobs (red) and $(B)$ labeled ocular dominance pattern (green) of the surviving eye were compared. Images were prepared following the method described in Figure 6 . $C$, In the color-coded map, CO blobs over the surviving eye's inputs are shown in yellow while the small amount of CO staining over the unlabeled regions is shown in red. Scale bar, $1 \mathrm{~mm}$.

the summed image (Fig. 9C), this confirms that the dense $\mathrm{CO}$ staining in layer IV after a total retinal lesion is in register with the regions subserved by the remaining eye's geniculocortical inputs (Fig. 9C).

\section{Discussion}

In this study we report patchy, nonuniform $\mathrm{CO}$ staining in the upper layers of cat visual cortex. This patchy pattern of dark $\mathrm{CO}$ staining is within the supragranular layers and bears a qualitative similarity to demonstrations of $\mathrm{CO}$ blobs in macaque visual cortex. In addition, the dark patches of $\mathrm{CO}$ staining in cat visual cortex appear to be functionally related to the modular organization of this area. Thus, based on similarities in both their appearance and their relationship to other features of the visual cortex, it seems prudent to apply the accepted nomenclature from the primate to describe the patterns of $\mathrm{CO}$ staining observed in these two species. Hence, we have referred to the dark patches of $\mathrm{CO}$ staining in cat visual cortex as blobs. This discovery lags by more than a decade the initial reports of $\mathrm{CO}$ blobs in primate V1 (Horton and Hubel, 1981), and subsequent attempts by numerous other groups (e.g., Wong-Riley, 1979; Horton, 1984; Price, 1985) to demonstrate the presence of CO blobs in nonprimate species.

At first glance, the present demonstration of $\mathrm{CO}$ blobs in the visual cortex of a nonprimate species may seem somewhat surprising, since until now it had been widely accepted that $\mathrm{CO}$ blobs represent a primate-specific feature of the visual cortex. However, using the standard $\mathrm{CO}$ histochemistry from Horton (1984) on sections from the unfolded and flattened visual cortex, blobs have proven to be a robust feature of layers II/III in adult cat visual cortex. The major technical difference in the present approach is that CO staining was examined throughout the complete tangential extent of area 17, in sections from unfolded and flattened cat cortex, whereas previous attempts had examined 


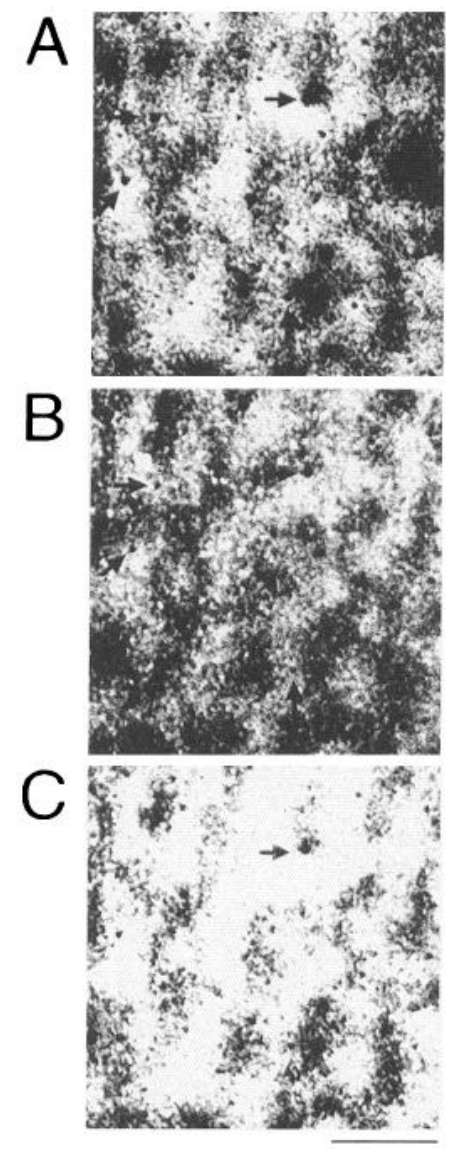

Figure 9. The relationship between $\mathrm{CO}$ staining and labeled ocular dominance patterns in adjacent sections from layer IV following monocular retinal lesion. A, A negative image of $\mathrm{CO}$ staining in layer IV after lesioning one eye's retina (white represents dense CO staining). $B$, Dark-field image of the ocular dominance pattern following injection of WGA-HRP into the surviving eye (white represents labeled inputs) $C$, The aligned images from $A$ and $B$ were added together to demonstrate that there is good correspondence between the regions of dense $\mathrm{CO}$ staining in layer IV after monocular retinal lesion and the surviving eye's inputs. The features of the pattern in $C$ resemble the anatomical features present in both $A$ and $B$.

radially cut sections. The finding that blobs are readily apparent in sections cut tangential to the pial surface in the cat affirms that this approach is more sensitive for revealing virtually any anatomical pattern arrayed parallel to the cortical laminae, especially if the pattern is of low contrast.

To our knowledge, no other histochemical staining method has revealed such a widespread, regular, patchy pattern that is an intrinsic feature of the supragranular layers in visual cortex of normally reared adult cats. Although, a recent report has described a patchy distribution of a molecularly distinct class of pyramidal neurons in layers II/III of adult cat visual cortex (Mareschal et al., 1994), and others have noted during development transiently patchy distributions of various markers, some in layers II/III, in the kitten visual cortex (Schoen et al., 1990; Dyck et al., 1993; Dyck and Cynader, 1993a), as well as patchy expression of the proto-oncogene c-fos (Beaver et al., 1992). Following our initial reports of CO blobs in adult cats (Murphy et al., 1990, 1991a,b), Dyck and Cynader (1993b) have observed patchy $\mathrm{CO}$ staining in the visual cortex of the kitten. Surprisingly, they have reported that at postnatal day $50 \mathrm{CO}$ staining is patchy in layer IV, in contrast to the present findings in adult cats, and previous data from primates (e.g., Horton, 1984), which show that $\mathrm{CO}$ staining is relatively uniform in layer IV and most patchy supragranularly.

Prior to the present study, nonuniform $\mathrm{CO}$ staining in adult cat visual cortex had been demonstrated in layer IV only following disruption of binocular vision (Wong-Riley, 1979; WongRiley and Carroll, 1984). There are several lines of reasoning to indicate that the variegated pattern of $\mathrm{CO}$ staining we find in layers II-III is not an inadvertent demonstration of the underlying ocular dominance pattern. First, all six cats studied were reared with normal binocular visual experience, and yet each clearly demonstrated blobs in both visual cortices. Furthermore, blobs were present in sections through layers II-III, whereas the staining pattern within layer IV was uniform (Fig. 4). Finally, if an abnormality associated with one eye had disrupted the pattern of cytochrome-oxidase staining in the cortex, then it also should have resulted in reduced staining of the layers of the dorsal lateral geniculate nucleus (dLGN) that receive their input from that eye (Wong-Riley and Riley, 1983; Kageyama and WongRiley, 1986). Examination of dLGN sections reacted for cytochrome oxidase revealed that the staining was of comparable density in the right- and left-eye layers of the normally reared cats (Fig. 3A), as compared with the CO staining of the dLGN layers following a monocular retinal lesion (Fig. 3B). All of these points indicate that there was no hidden abnormality in the primary visual pathway of these cats that could have caused a patchy pattern of cytochrome-oxidase staining in layers II-III. Evidently, $\mathrm{CO}$ blobs are a normal feature of cat visual cortex.

In the monkey, although the blobs are superficial to the zone of densest geniculocortical input, the blobs do receive some direct thalamic input from the intercalated layers of the dLGN (Livingstone and Hubel, 1982; Fitzpatrick et al., 1983; Horton, 1984; Wong-Riley and Carroll, 1984; Lachica and Casagrande, 1992). In the primate visual cortex there is a correlation between the arrangement of geniculocortical inputs and dense CO staining: it is precisely those regions that receive direct thalamic input (the blobs, layers IVA, IVC, and VI) where CO staining is most dense. The radial relationship between geniculocortical inputs and the pattern of CO staining in cat visual cortex appears similar to that described for the primate and is consistent with the possibility that blobs may receive some direct thalamic input. Geniculocortical input to the lower tier of layers II-III in the cat is from the C laminae of the dLGN and injection of an anterograde tracer into the $\mathrm{C}$ laminae, such that each eye's laminae are labeled, results in patchy labeling in the visual cortex with an interpatch spacing of approximately $500 \mu \mathrm{m}$ (LeVay and Gilbert, 1976; Leventhal, 1979). Thus, the radial and tangential pattern of $\mathrm{CO}$ staining in cat visual cortex is consistent with the notion that dense $\mathrm{CO}$ staining may be related to direct input from the dLGN. In addition, the CO blobs in cat visual cortex are most distinct in the region representing the area centralis, where physiological investigations have found the highest proportion of monocularly driven cells (Albus, 1975), and anatomical studies have demonstrated the most distinct pattern of ocular dominance columns (Anderson et al., 1988).

\section{Methodological considerations}

Since the introduction of techniques for flattening the cortical mantle to examine patterns arrayed tangential to the cortical laminae (Woolsey and Van der Loos, 1970; Welker and Woolsey, 1974), the use of this type of preparation has grown steadily. When applied to the study of gyrencephalic brains, however, 
there is the potential that less than optimal unfolding and flattening strategies may introduce physical distortions or possibly even artifactual histological results. The procedure used in this study for unfolding and flattening the visual cortex, developed by Olavarria and Van Sluyters (1985), produces a well-fixed sheet of cortical gray matter that maintains its cellular integrity (see Fig. 5 in Olavarria and Van Sluyters, 1985) and is resilient to the flattening process. As Olavarria and Van Sluyters indi cated, once the white matter is removed and the sulci are opened, the shape of area 17 is similar to the hull of a ship, and this significant intrinsic curvature must be relieved prior to flattening to prevent distortions of area 17. Following the lessons learned by cartographers, who sought to render a distortion-free flat map by introducing a series of discontinuities that relieved the intrinsic curvature of the globe, the visual cortex is flattened by introducing a medial and a lateral cut in the tissue (see Fig. $1 B$ in Olavarria and Van Sluyters, 1985). Although this procedure does result in a discontinuity within area 17 , without cuts sufficient to relieve the intrinsic curvature, the process of flattening will result in one of two undesired outcomes. In the case where the visual cortex is adequately fixed, during flattening it will tear at the point of maximum curvature (evidence of this can been seen in Fig. 1A). If the cortex is unfixed, or only very lightly fixed, so that it remains extremely pliable, then instead of tearing, it may relieve its intrinsic curvature by undergoing differential stretching, compression, and shearing, which must result in distortion of the tissue. Since the cortical mantle is a three-dimensional slab of tissue, this distortion could be very complex. The potential for nonoptimal flattening to distort the tissue has obvious implications for quantitative analyses of tangentially arrayed patterns. Another scrious concern is the possibility that forcing a piece of unfixed or lightly fixed tissue to lie flat may disrupt its laminar and/or cellular integrity, thereby adversely affecting subsequent histological analyses. Given these obvious risks, we chose a procedure where the cortical gray matter is firm and cuts are made in the cortical tissue to relieve the intrinsic curvature of this region, rather than a "flat-mount" technique on lightly or unfixed tissue that does not introduce cuts into area 17.

In light of the above discussion, a potential, albeit unlikely, concern is that this demonstration of blobs in cat visual cortex could somehow be an artifact of the unfolding and flattening procedure. This seems highly improbable for a number of reasons. First, blobs were found only in the supragranular layers in area 17 -the same area and layers where they are found in primates. In addition, the laminar location and 2D pattern of blobs was similar for all cases examined (see Fig. 2). Finally, when we have examined the pattern of $\mathrm{CO}$ staining in macaque visual cortex using the same perfusion, unfolding, flattening, and $\mathrm{CO}-$ staining techniques described in this study (K. Murphy, unpublished observations), the blob pattern, the thick and thin stripes, and the laminar variations in CO staining are the same as those previously reported (e.g., Horton, 1984). Thus, the logical conclusion from this evidence is that $\mathrm{CO}$ blobs are a robust feature of the cat visual cortex.

\section{Relationship between blobs and ocular dominance columns}

The significance of $\mathrm{CO}$ blobs in primates for understanding the organization of the visual cortex follows from their functional relationship with other anatomical and physiological features of $\mathrm{V} 1$. To begin to address this issue in the cat visual cortex, we examined the relationship of $\mathrm{CO}$ blobs to ocular dominance col- umns and found evidence that blobs are functionally related with the underlying ocular dominance columns. Initial analysis of the position of blobs with respect to the ocular dominance columns revealed that there are blobs over each eye's columns; thus, blobs do not simply reflect one eye's columns. In addition, the location of some blobs in cat visual cortex is not above the center of a labeled ocular dominance domain, which raises the possibility that they may not be functionally eye specific. This arrangement contrasts with that in monkey visual cortex, where individual blobs are centered upon and confined entirely within the boundaries of an underlying ocular dominance column (Fitzpatrick and Diamond, 1980; Hendrickson et al., 1981; Horton, 1984). At first glance, these spatial differences might give the impression that, unlike in the macaque, in the cat the distribution of blobs is functionally unrelated to ocular dominance columns. However, there are at least two points to consider in interpreting the present analysis of the organization of blobs in the cat. First, the apparent diameter of the blobs in cat visual cortex in comparison to the width of the labeled ocular dominance columns is greater than that relationship in monkeys. Thus, it would not be uncommon for the edges of a blob to overlap the adjacent ocular dominance domains, even if the blob was centered upon an unlabeled region. Second, in macaque visual cortex, the geniculostriate afferents are strictly segregated, with virtually no overlap of the inputs from the two eyes (Hubel and Wiesel, 1977; Blasdel and Fitzpatrick, 1984; LeVay et al., 1985). In the cat, on the other hand, there is significant overlap of the inputs from the two eyes within layer IV (Shatz and Stryker, 1978), resulting in much less area that is strictly monocular than is the case in the macaque. The regions labeled in cat visual cortex following an eye injection indicate both the zones where only the injected eye has inputs, as well as the regions where it overlaps with the fellow eye. Only the unlabeled regions reflect the truly monocular aspects of ocular dominance columns in the cat. Consequently, a simple analysis of the position of blobs above the ocular dominance pattern may fail to reveal if there is a functional link between these two features. To examine the functional relationship between blobs and ocular dominance in the cat, in the same manner as has been done for the monkey, it is necessary to identify and manipulate the strictly monocular aspects of the ocular dominance columns, then to compare those monocularity maps with the array of blobs. To the extent that such an approach reveals a relationship between blobs and monocular regions of the ocular dominance pattern, it provides support for the notion that the functional organization of the blobs is similar in cats and monkeys.

The question of a functional relationship between blobs and ocular dominance was addressed in the present study by silencing the activity of one eye, the manipulation used previously to make this functional link in monkeys. The results of the present experiments indicate that $\mathrm{CO}$ blobs in cat visual cortex are related to specific aspects of the ocular dominance columns. First, in normally reared cats there are some blobs over the truly monocular regions of layer IV-those areas not labeled by an eye injection. Second, when the inputs from one eye are silenced, there is a complete loss of blobs over the regions aligned with the monocular domains of the enucleated eye. Thus, the presence of CO blobs in layers II/III of adult cat visual cortex is contingent upon the presence of active geniculocortical inputs (from either the $\mathrm{A}, \mathrm{A} 1$, or $\mathrm{C}$ laminae), the same inputs that form the anatomical basis of ocular dominance columns. However, the pattern of CO staining in layers II/III does not simply reflect the 
pattern of active geniculocortical inputs within layer IV, since the regions of dark $\mathrm{CO}$ staining in the supragranular layers remain patchy and do not become as extensive as the underlying inputs from the surviving eye.

\section{Cortical modularily}

The demonstration of blobs in the visual cortex of the cat strengthens the evidence for a modular organization within this area. It also raises the intriguing possibility that streams of visual processing, similar to those reported for the primate visual system (Zeki, 1975; Livingstone and Hubel, 1983, 1984a; Shipp and Zeki, 1985; DeYoe and Van Essen, 1985, 1988; Van Essen et al., 1992), may have parallels in the visual systems of these other mammals (Shipp and Grant, 1991; Boyd and Matsubara, 1992). In addition, it is possible that by employing the approach of the present study, blobs may be revealed in the visual cortex of other mammals. Clearly, CO blobs can no longer be referred to as a "primate-specific" feature of the visual system, and their role as a more general characteristic of mammalian cortical organization must now be considered. The compelling qualitative similarities in the appearance of blobs in cat and macaque visual cortex underscore the idea that the functional organization of this area in these two species may have more in common than was previously proposed.

Exactly what the functional significance of the blobs in cat visual cortex is, and how closely the responses of cells within these blobs will match those reported for blob-cells in primate cortex are currently unexplored questions. Blob-cells in primate visual cortex have been reported to exhibit certain characteristic physiological selectivities for color, orientation, and spatial frequency, in addition to their relationship with ocular dominance. The next stage in understanding the significance of blobs in these other mammals will involve exploring the physiological characteristics of blob and interblob cells. It is known that cats have relatively poorly developed color vision (e.g., Weinrich and Zrenner, 1983); consequently, it seems likely that blob cells in cat visual cortex may be similar to those in nocturnal primates (DeBruyn et al., 1993) and not exhibit color selectivity similar to that observed in diurnal primate species. On the other hand, physiological and anatomical information about the organization of the orientation map makes it a possible candidate. Orientation selectivity is well developed and its tangential arrangement is known to be periodic (Schoppman and Stryker, 1981; Singer, 1981; Bonhoeffer and Grinvald, 1991, 1993). Furthermore, the patchy intracortical connections in the supragranular layers of cat cortex (Gilbert and Wiesel, 1979, 1983; Callaway and Katz, 1990; Kalz and Callaway, 1992) are known to link like orientations (Gilbert and Wiesel, 1989; T'so et al., 1986). The blobs are interconnected in primate visual cortex (Livingstone and Hubel, 1984b), raising the possibility that the horizontal connections in cat visual cortex also may represent a blob-specific network that links regions with similar orientation selectivity.

In addition to studying the tangential pattern of $\mathrm{CO}$ staining in cat visual cortex, we, as well as others, have made preliminary investigations of a second species, the ferret (Cresho et al., 1992; Murphy and Stryker, unpublished observations). Sections through surpagranular layers of unfolded and flattened ferret visual cortex revealed patches of dark CO staining regularly spaced within area 17 . These patches may relate to features of the ferret visual cortex that are arrayed in a periodic fashion (Redies et al., 1990). The dark patches of CO staining in the ferret were qualitatively similar to those observed in cat visual cortex, although the contrast between blob and interblob regions appeared somewhat less in the ferret. Relative to the tangential area of visual cortex the blobs appear large, so that in comparison with the number of blobs in cat or macaque, there are fewer blobs in ferret visual cortex. This extends the observation made previously by Horton (1984) for primates. The size and number of blobs appears to vary considerably across species.

Recent controversy over the degree of segregation of physiological properties into blob and interblob zones in primate cortex (see Merigan and Maunsell, 1993) has raised concerns that the importance of the blobs may not be as great as originally proposed. However, a recent hypothesis about the functional significance of $\mathrm{CO}$ blobs that focuses upon the style of computations possible with metabolically more active neurons provides a different perspective, and a possible species-independent function for blob-cells that goes beyond limits set by visual niche differences (Allman and Zucker, 1990). After all, the patchy CO staining observed in supragranular layers of the mammalian visual cortex is an indication that the metabolic activity across its tangential extent is not uniform. How metabolic activity becomes organized in this periodic fashion has remained a largely unstudied question. One thing that is clear is that the development of CO blobs in primate visual cortex is not strictly dependent upon retinal activity, since blobs are present even after removal of both retinae very early in gestation (Kuljis and Rakic, 1990). Furthermore, hlobs are present even when the generation of layer IV neurons is disrupted resulting in a failure of geniculocortical afferents to segregate into ocular dominance columns (Kind et al., 1993). Perhaps it is wise to begin to view the significance of the blobs for understanding the organization of the visual cortex, and ultimately vision, in a manner that is consistent with these results-the patchy organization of CO blobs appears to reflect an intrinsic periodicity of the mammalian visual cortex that is independent of certain developmental processes that rely upon retinally based activity (Jones et al., 1991).

Our demonstration of $\mathrm{CO}$ blobs in cat visual cortex indicates that they are a more ubiquitous feature of mammalian cortical organization than was previously believed and provides a new model system for comparative investigations of their significance for visual cortical organization. At this stage, the regular, patchy nature of $\mathrm{CO}$ blobs in the visual cortex of the cat reaffirms the general notion of modularity within the mammalian visual cortex and underscores the importance of species-independent models of functional organization within the visual system.

\section{References}

Albus K (1975) Predominance of monocularly driven cells in the projection area of the central visual field in cat's striate cortex. Brain Res 89:341-347.

Allman J, Zucker S (1990) Cytochrome oxidase and functional coding in primate striate cortex a hypothesis. Cold Spring Harbor Symp Quant Biol 55:979-982.

Anderson PA, Olavarria J, Van Sluyters RC (1988) The overall pattern of ocular dominance bands in cat visual cortex. J Neurosci 8:21832200.

Beaver C, Murphy KM, Mitchell DE (1992) Patchy expression of c-fos in kitten visual cortex. Soc Neurosci Abstr, 18:1307.

Bonhoeffer T, Grinvald A (1991) Iso-orientation domains in cat visual cortex are arranged in pinwheel-like patterns. Nature 353:429-431.

Bonhoeffer T, Grinvald A (1993) The layout of iso-orientation domains in area 18 of cat visual cortex: optical imaging reveals a pinwheellike organization. J Neurosci 13:4157-4180.

Boyd JD, Matsubara JA (1992) Segregated processing streams in cat visual cortex? Relationship of patchy connectivity to an extrastriate 
area, cytochrome-oxidase staining, and local connections. Soc Neurosci Abstr 22:298.

Callaway EM, Katz LC (1990) Emergence and refinement of clustered horizontal connection in cat striate cortex. J Neurosci 10:1134-1153

Cresho HS, Rasco LM, Rose GH, Condo GJ (1992) Blob-like pattern of cytochrome oxidase staining in ferret visual cortex. Soc Neurosci Abstr 18:298.

DeBruyn EJ, Casagrande VA, Beck PD, Bonds AB (1993) Visual resolution and sensitivity of single cells in the primary visual cortex (V1) of a nocturnal primate (bush baby): correlations with cortical layers and cytochrome oxidase patterns. J Neurophysiol 69:3-18.

DeYoe EA, Van Essen DC (1985) Segregation of efferent connections and receptive field properties in visual area V2 of the macaque. Nature 317:58-61.

DeYoe EA, Van Essen DC (1988) Concurrent processing streams in monkey visual cortex. Trends Neurosci 5:219-226.

Dyck R, Beaulieu C, Cynader M (1993) Histochemical localization of synaptic zinc in the developing cat visual cortex. J Comp Neurol 329:53-67.

Dyck RH, Cynader MS (1993a) Autoradiographic localization of serotonin receptor subtypes in cat visual cortex: transient regional, laminar, and columnar distributions during postnatal development. J Neurosci 13:4316-4338.

Dyck RH, Cynader MS (1993b) An interdigitated columnar mosaic of cytochrome oxidase, zinc, and neurotransmitter-related molecules in cat and monkey visual cortex. Proc Natl Acad Sci USA 90:90669069.

Fitzpatrick D, Diamond IT (1980) Distribution of acetylcholinesterase in the geniculostriate system of Galago senegalensis and Aotus trivirgatus: evidence for the origin of the reaction product in the lateral geniculate body. J Comp Neurol 194:703-719.

Fitzpatrick D, Itoh K, Diamond I'T' (1983) The laminar organization of the lateral geniculate body and the striate cortex in the squirrel monkey (Saimiri sciureus). J Neurosci 3:673-702.

Gibson AR, Hansma DI, Houk JC, Robinson FR (1984) A sensitive low artifact TMB procedure for the demonstration of WGA-HRP in the CNS. Brain Res 298:235-241.

Gilbert CD, Wiesel TN (1979) Morphology and intracortical projections of functionally characterised neurones in the cat visual cortex. Nature 280:120-125.

Gilbert CD, Wiesel TN (1983) Clustered intrinsic connections in cat visual cortex. J Neurosci 3:1116-1133

Hendrickson AE, Hunt SP, Wu JY (1981) Immunocytochemical localization of glutamic acid decarboxylase in monkey striate cortex. Nature 292:605-607.

Horton JC (1984) Cytochrome oxidase patches: a new cytoarchitectonic feature of monkey visual cortex. Philos Trans R Soc Lond [Biol] 304:199-253.

Horton JC, Hubel DH (1981) Regular patchy distribution of cytochrome oxidase staining in primary visual cortex of macaque monkey. Nalure 292:762-764

Hubel DH, Livingstone MS (1987) Segregation of form, color, and stereopsis in primate area 18. J Neurosci 7:3378-3415.

Hubel DH, Wiesel TN (1974) Uniformity of monkey striate cortex: a parallel relationship between field size, scatter, and magnification factor. J Comp Neurol 158:295-305.

Jones DG, Van Sluyters RC, Murphy KM (1991) A computational model for the overall pattern of cortical ocular dominance. $J$ Neurosci $11: 3794-3807$.

Kageyama GH, Wong-Riley M (1986) Differential effect of visual deprivation on cytochrome oxidase levels in major cell classes of the cal LGN. J Comp Neurol 246:212-237.

Katz LC, Callaway EM (1992) Development of local circuits in mammalian visual cortex. Annu Rev Neurosci 15:31-56

Kind P, Murphy KM, Sengpiel F, Blakemore C (1993) Disruption of generation of layer IV in cat area 17 retards geniculate axon segregation but not the emergence of cytochrome-oxidase blobs. Soc Neurosci Abstr 19:176.

Kuljis RO, Rakic P (1990) Hypercolumns in primate visual cortex can develop in the absence of cucs from photoreceptors. Proc Natl Acad Sci USA 87:5303-5306.

Lachica EA, Casagrande VA (1992) Direct W-like geniculate projection to the cytochrome oxidase $(\mathrm{CO})$ blobs in primate visual cortexaxon morphology. J Comp Neurol 319:141-158.
LeVay S, Gilbert CD (1976) Laminar patterns of geniculocortical projection in the cat. Brain Res 113:1-19.

Leventhal AG (1979) Evidence that the different classes of relay cells of the cat's lateral geniculate nucleus terminate in different layers of the striate cortex. Exp Brain Res 37:349-372.

Livingstone MS, Hubel DH (1982) Thalamic inputs to cytochrome oxidase-rich regions in monkey visual cortex. Proc Natl Acad Sci USA 79:6098-6101

Livingstone MS, Hubel DH (1983) Specificity of cortico-cortical connections in monkey visual system. Nature 304:531-534.

Livingstone MS, Hubel DH (1984a) Anatomy and physiology of a color system in the primate visual cortex. J Neurosci 4:309-356.

Livingstone MS, Hubel DH (1984b) Specificity of intrinsic connections in primate primary visual cortex. J Neurosci 4:2830-2835.

Livingstone MS, Hubel DH (1987a) Connections between layer 4B of area 17 and the thick cytochrome oxidase stripes of area 18 in the squirrel monkey. J Neurosci 7:3371-3377.

Livingstone MS, Hubel DH (1987b) Psychophysical evidence for separate channels for the perception of form, color, movement, and depth. J Neurosci 7:3416-3468.

Marcschal I, Joncs DG, Murphy KM (1994) Tangential distribution of pyramidal neurons in cat areas 17 and 18. Invest Ophthalmol Vis Sci [Suppl] 35:1971.

Merigan WH, Maunsell JH (1993) How parallel are the primate visual pathways? Annu Rev Neurosci 16:369-402.

Murphy KM, Jones DG, Van Sluyters RC (1989) Analysis of the overall pattern of ocular dominance in normal and visually deprived cats. Invest Ophthalmol Vis Sci [Suppl] 30:30.

Murphy KM, Van Sluyters RC, Jones DG (1990) Cytochrome-oxidase activity in cat visual cortex: is it periodic? Soc Neurosei Abstr 16: 292.

Murphy KM, Van Sluyters RC, Jones DG (1991a) Cytochrome-oxidase blobs in cat visual cortex. Invest Ophthalmol Vis Sci [Suppl] 32: 1116.

Murphy KM, Van Sluyters RC, Jones DG (1991b) Analysis of the tangential arrangement of cytochrome-oxidase blobs in cat visual cortex. Soc Neurosci Abstr 17:1088.

Price DJ (1985) Patterns of cytochrome oxidase activity in areas 17, 18 and 19 of the visual cortex of cats and kittens. Exp Brain Res 58: $125-133$

Olavarria J, Van Sluyters RC (1985) Unfolding and flattening the cortex of gyrencephalic brains. J Neurosci Methods 15:191-202.

Redies C, Diksic M, Riml H (1990) Functional organization in the ferret visual cortex: a double label 2-deoxyglucose study. J Neurosci 10:2791-803

Schoen SW, Leutenecker B, Kreutzberg GW, Singer W (1990) Ocular dominance plasticity and developmental changes of $5^{\prime}$-nucleotidase distributions in the kitten visual cortex. J Comp Neurol 69:3-18.

Schoppmann A, Stryker MP (1981) Physiological evidence that the 2-deoxyglucose method reveals orientation columns in cat visual cortex. Nature 293:574-576.

Shipp S, Grant S (1991) Organization of reciprocal connections between area 17 and the lateral suprasylvian area of cat visual cortex. Vis Neurosci 6:339-35.5.

Shipp S, Zeki S (1985) Segregation of pathways leading from area V2 to areas V4 and V5 of macaque monkey visual cortex. Nature 315: $322-325$.

Singer W (1981) Topographic organization of orientation columns in the cat visual cortex. A deoxyglucose study. Exp Brain Res 44:431436.

Ts'o DY, Gilbert CD, Wiesel TN (1986) Relationships between horizontal interactions and functional architecture in cat striate cortex as revealed by cross-correlation analysis. J Neurosci 6:1160-1170.

Van Essen DC, Anderson CH, Felleman DJ (1992) Information processing in the primate visual system: an integrated systems perspective. Science 255:419-423

Welker C, Woolsey TA (1974) Structure of layer IV in the somatosensory neocortex of the rat: description and comparison with the mouse. J Comp Neurol 158:437-453.

Wong-Riley M (1979) Changes in the visual system of monocularly sutured or enucleated cat demonstrable with cytochrome oxidase histochemistry. Brain Res 171:11-28.

Wong-Riley M, Carroll EW (1984) Effect of impulse blockage on cytochrome oxidase activity in monkey visual system. Nature 307:262264. 
Wong-Riley M, Riley DA (1983) The effect of impulse blockage on cytochrome oxidase activity in the cat visual system. Brain Res 261: 185-193.

Woolsey TA, Van der Loos H (1970) The structural organization of layer IV in the somatosensory region (SI) of mouse cerebral cortex.
The description of a cortical field composed of discrete cytoarchitectonic units. Brain Res 17:205-242.

Zeki SM (1976) The functional organization of projections from striate to prestriate visual cortex in the rhesus monkey. Cold Spring Harhor Symp Quantitat Biol 40:591-600. 